designated course of psychotherapy of a particular kind. However, from the moment of referral, to selection, engagement, working through, and finally termination of treatment, these clients represent a progressively filtered and highly selected subgroup. While they may, in a rather self-fulfilling way, fit the Western template of suitable candidates for psychotherapy, they would not allow one to determine whether more or fewer non-Western people, compared with Western subjects, generally respond to 'Western' psychotherapy. From a 'new' crosscultural point of view, it may paradoxically be of great interest to study the characteristics of the reject or dropout cases, and find out in what other ways they can be engaged in a mutually agreed form of healing, or to whom they go for alternative forms of help and why. Unfortunately, these subjects seem to be ignored in 'cross-cultural' research on psychotherapy.

The thesis that 'Western' psychotherapy is (not) applicable for non-Western people therefore involves two intrinsically complex factors, non-Western people and 'Western' psychotherapy, and is difficult to test empirically. Psychotherapy has been said to lie in the realms of rhetoric and hermeneutics. Its credibility is enhanced by invoking the prestige of Western science but, paradoxically, is not scientifically ascertainable (Frank, 1988). If psychotherapists seriously want to study the question of cross-cultural application, they would have to move beyond value-laden personal experience and single case reports to examine, preferably with inter-disciplinary efforts and culturally relevant models, specific dimensions of psychotherapy in relation to specific subgroups of non-Western people. However, the justification for such an endeavour may deplorably be questioned by many therapists themselves and the required crosscultural methods and instruments are far from being developed at this stage. Until then, discussion on the question of cross-cultural applicability of psychotherapy seems to be mainly an emotional one.

FrANK, J. (1988) Specific and non-specific factors in psychotherapy. Current Opinion in Psychiatry, 1, 289-292.

Mezzich, J. E., FAbregA, H. JR. \& Kletnman, A. (1992) Cultural validity and DSM-IV. Journal of Nervous and Mental Disease, $180,4$.

WANG, M. C. (1986) Psychological Treatment in Traditional Medicine. Chungking, China: Chungking Publishing Company.

Department of Psychiatry

SING LEE

Chinese University of Hong Kong

Shatin

Hong Kong

\section{Psychological outcome of abortion}

SIR: In an era when the expression of strident and polemical viewpoints concerning all aspects of abortion has become the norm, cool-headed attempts at evaluating the psychological outcome for the women concerned are greatly needed. As such, the paper by Zolese \& Blacker (Journal, June 1992, 160, 742-749) is to be welcomed.

We feel, however, that the authors could have done more to highlight the difficulties in conducting research in this area, particularly regarding the choice of control subjects.

When the goal is simply to identify a population at risk of psychological dysfunction it is sufficient to compare recipients of abortion with non-pregnant women of similar age. To isolate the effects, both mental and physical, of therapeutic abortion is rather more difficult, as there is no comparison group where confounding factors are small.

A relevant question here is "what are the psychological consequences of denying an abortion?". Thus one comparison group might be women whose request for a termination is refused. Although in a country where termination of pregnancy is widely available this group might be more or less psychologically disturbed, the comparison seems a worthwhile one since this group would represent the only possible alternative outcome of an unwanted pregnancy. The interested reader is referred to an earlier review (Handy, 1982) for a fuller discussion of these issues.

Handy, J. A. (1982) Psychological and social aspects of induced abortion. British Journal of Clinical Psychology, 21, 29-41.

The Maudsley Hospital

Denmark Hill

London SE5 8 AZ

\section{'Socrates' symptom'}

SIR: Förstl (Journal, June 1992, 160, 868-869) reminds us of Socrates' experiences and behaviour and suggests the term 'Socrates' symptom' for the combination of auditory hallucination and cataplexia-like symptoms occurring in stressful situations. Although being a soldier in a time of war is certainly stressful, Socrates seems the least likely person to become disturbed by this, as he was renowned as a man indifferent to physical pain and hardship (Xenophon, Symposium) and indeed accepted his own death 'philosophically'. 